\title{
Spectral Integration of Microstructure and Design
}

\author{
Brent L. Adams \\ b_I_adams@byu.edu \\ B. Henrie \\ M. Lyon \\ H. Garmestani \\ Surya R. Kalidindi
}

Follow this and additional works at: https://scholarsarchive.byu.edu/facpub

Part of the Mechanical Engineering Commons

Original Publication Citation

Materials Science Forum Vols. 48-412 (22) pp. 493-498

\section{BYU ScholarsArchive Citation}

Adams, Brent L.; Henrie, B.; Lyon, M.; Garmestani, H.; and Kalidindi, Surya R., "Spectral Integration of Microstructure and Design" (2002). Faculty Publications. 1066.

https://scholarsarchive.byu.edu/facpub/1066

This Peer-Reviewed Article is brought to you for free and open access by BYU ScholarsArchive. It has been accepted for inclusion in Faculty Publications by an authorized administrator of BYU ScholarsArchive. For more information, please contact ellen_amatangelo@byu.edu. 


\title{
Spectral Integration of Microstructure and Design
}

\author{
B.L. Adams ${ }^{1}$, M. Lyon ${ }^{1}$, B. Henrie', S.R. Kalidindi ${ }^{2}$ and H. Garmestani ${ }^{3}$ \\ 1 Department of Mechanical Engineering, Brigham Young University, \\ 435 Crabtree Technology Building, Provo, UT 84602, USA \\ ${ }^{2}$ Department of Materials Engineering, Drexel University, \\ $32^{\text {nd }}$ and Chestnut Streets, Philadelphia, PA 19104, USA \\ ${ }^{3}$ Department of Mechanical Engineering, FAMU-FSU College of Engineering, \\ 2525 Pottsdamer Street, Tallahassee, FL 32310, USA
}

Keywords: Elasticity, Mechanical Design, Microstructure, Plasticity, Series Method, Texture

Abstract. Mechanical design can be conducted in a framework where consideration of microstructure as a continuous design variable is facilitated by the use of a Fourier space. Selection of the mechanical framework for the problem (e.g., mechanical constitutive model and homogenization relations) dictates the dimensionality of the pertinent microstructure representation. Microstructure is comprised of basic elements that belong to the local state space. Local state includes crystallographic phase and orientation, and other parameters such as composition. The local state space is transformed into an isomorphic set in Fourier space. The universe of pertinent microstructures is found to be the convex hull of the local state space in the Fourier space, and is named the microstructure hull. Bounds on material properties are represented by one or more families of bounding hyper-surfaces (often hyper-planes) of finite dimension that intersect the microstructure hull. Consideration of the full range of these hypersurfaces gives rise to properties closures, representing the full range of combined properties that are predicted to be possible by considering the entire microstructure hull. We describe how properties closures can be introduced into design optimization systems, thereby introducing microstructure as a continuous variable in the mechanical design methodology for highlyconstrained systems. Also, we describe the challenges and possibilities for extending the methodology to processing to achieve the prescribed optimal microstructures.

\section{Introduction}

In highly-constrained (threshold) design problems it is rare that performance would depend upon any single material property in isolation from all others. Far more commonly, several properties are manifestly important, to varying degrees, to design performance. It is also typical that the anisotropic nature of properties must be fully exploited where possible. Here we describe a new approach to highly-constrained design wherein the quantitative description of microstructure and associated microstructure-properties relationships are completely described in a Fourier space. The main idea behind the work was first described for a problem of low-dimension (second rank tensors of orthorhombic materials possessing a fiber texture) by Bunge [1]. Extensions of the idea to higherdimensions and to combinations of properties was described in recent publications by Adams et al. [2,3]. The concept of properties closures was described very briefly by Adams et al. [4], and is treated in more detail in this paper in the special case where lower-bounds are of interest. Specifically, we focus upon a lower-bound on the elastic compliance $S_{1111}^{*}$, and a (pseudo) lower-bound on the yield strength $\sigma_{11}^{y}$ in single-phase fcc cubic-orthorhombic polycrystals. The methodology described, 
however, is applicable to all possible combinations of elastic-plastic properties in all types of heterogeneous materials.

\section{Microstructure Design for Elastic-Plastic Properties}

A basic problem of mechanical design is often one of achieving a high degree of stiffness or compliance in one or more components of the elasticity tensor, without plastic yielding. Here we illustrate the basic methodology by describing the steps required to obtain the (combined) properties closure associated with $S_{1111}^{*}$ vs. $\sigma_{11}^{y}$. The closure consists of the set of all possible combinations of the two properties, as predicted by the selected microstructure-properties relationships, and the microstructure-hull that describes all possible microstructures. The basic premise behind the development is that salient local properties are dependent upon a small number of local state variables that are known to the observer. Effective (macroscopic) properties are estimated by the application of suitable homogenization relationships that link the spatial distribution of local properties to the corresponding effective ones. Properties closures then follow by systematic investigation into the classes of microstructures that are predicted to obtain the same properties. The basic methodology is now described in greater detail.

\section{Specification of Local State Variables}

For our purposes here, local state variables include lattice orientation, $g$, and a single scalar parameter, $c$, that may represent variables like composition, reference shear stress for dislocation slip, or others. Local state space, $H$, shall consist of all possible (ordered) sets of local state variables $h=(g, c) \in H$. Extensions of the methodology to include additional local state variables is straightforward.

\section{Selection of Homogenization Relationships}

Appropriate homogenization relationships relating the elastic and plastic properties of interest to the distribution of local state variables in the materials of interest can be selected. Here, for simplicity, first-order bounds on the effective elastic compliance, $S_{1111}^{*}$, are obtained from the Hill-Paul bounding theory $[5,6]$, which can be expressed in the form

$$
\langle C\rangle_{1111}^{-1} \leq S_{1111}^{*} \leq\left\langle S_{1111}(h)\right\rangle,
$$

where $\langle()\rangle$ denotes the volume average of () . Our primary interest shall be in the left-hand side of this relationship, which describes the lower-bound in terms of components of the inverse of the average stiffness tensor.

For yielding we choose the Sachs' model [7], which lies everywhere within the Taylor upper-bound model. The Cauchy stress, $\sigma$, in any crystallite of the composite material is assumed to be a simple scalar multiplier of a fixed unit stress $\hat{o}$ :

$$
\sigma=\lambda \partial \text {. }
$$

The unit stress is taken to be constant among all components of the material. The scaling parameter is dependent upon the local state $h$ in the crystallite, which is determined in a particular way. It is considered that plastic yielding occurs when the resolved shear stress on any particular slip system of the crystallite reaches a critical level (i.e., Schmid's law). For a crystallite with slip system $s$ characterized by the unit slip plane normal $\hat{n}^{(s)}$ and the unit slip direction $\hat{b}^{(s)}$, the resolved shear 
stress is $\tau^{(s)}=\hat{n}^{(s)} \lambda \hat{o} \hat{b}^{(s)}$. It is convenient to write this expression in terms of the fundamental slip tensor $\mu^{(s)}$, as

$$
\tau^{(s)}=\mu^{(s)} \lambda \sigma^{2}
$$

where

$$
\mu_{i j}^{(s)}=\frac{1}{2}\left(b_{i}^{(s)} n_{j}^{(s)}+b_{j}^{(s)} n_{i}^{(s)}\right) .
$$

Next, let $\tau_{c}^{(s)}$ denote the critical resolved shear stress at which slip on slip system $s$ occurs in the crystallite. This will occur at different levels of the scaling parameter $\lambda^{(s)}$ for each slip system. Hence the yielding criterion for slip is just

$$
\tau^{(s)}=\tau_{c}^{(s)}
$$

According to Sachs, yielding occurs at a given material point of local state $h$ when the first slip system is activated upon loading. Thus, if $s^{*}$ denotes the particular slip system that is first activated, $\lambda^{\left(s^{*}\right)}$ is the minimum among the scaling factors among the available set of slip systems $\left\{\lambda^{(s)}\right\}$. For convenience in notation we hereafter set the Sachs' local stress estimate, $\sigma=\sigma(h)$, to be

$$
\sigma(h)=\lambda^{\left(s^{*}\right)} \partial=\lambda * \partial,
$$

where it is understood that $\lambda^{*}=\lambda^{*}(h, \hat{\sigma})=\lambda^{\left(s^{*}\right)}$ derives its dependence upon local state $h$ through $\tau_{\mathrm{r}}^{(s)}$ and $\mu^{(s)}$. Sachs' model thus estimates the effective yield stress, $\sigma_{11}^{y}$, to be the volume average of $\lambda^{*} \hat{o}_{11}$ :

$\sigma_{11}^{y} \approx\langle\lambda *\rangle \sigma_{11}$.

Eqs. $1 \& 7$ specify the relationships between microstructure and effective (elastic-plastic) properties.

\section{Homogenization Relationships in the Fourier Space}

Eqs. 1 \& 7 require only volume fraction information on the local state space, $H$. This is typical of first-order homogenization relations. To implement the bounds we require a distribution function of the form $f: H \rightarrow \mathfrak{R}^{+}$, describing the volume fraction of material associated with local state $h$, say $d V / V=f(h) d h$. Any such distribution function can be specified as an infinite series of orthonormal basis functions on the local state space; thus

$$
f(h)=\sum^{\infty} \sum^{M(l)} \sum^{+l} \sum_{p}^{P} F_{l}^{\mu n} \dot{T}_{l}^{\mu n}(g) \chi_{p}(c)
$$

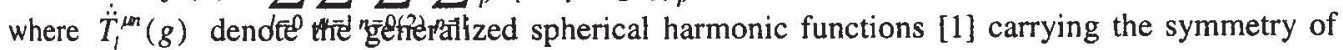
the lattice, $\chi_{p}(c)$ are defined to be piecewise constant functions [8] defined on subintervals of the range of $c$ (as enumerated by the index $p$ ), and ${ }_{p} F_{l}^{\mu n}$ are the Fourier coefficients that define the microstructure represented by the function $f$.

The left hand side of Eq. 1 requires the volume-average stiffness tensor, which average can be represented by the same orthonormal basis. Upon convolution with the distribution function, this average becomes

$$
\left\langle C_{i j k l}\right\rangle=\sum_{l=0}^{4} \sum_{n=0(2)}^{+l} \sum_{p=1}^{P} F_{l}^{l n}{ }_{p} E_{l(i j k l)}^{1 n}
$$


where the coefficients of the components of the average stiffness tensor are ${ }_{p} E_{l(i j k l)}^{\mu n}$. To form the lower bound on the left of relation Eq. 1 requires the inversion of Eq. 9 using established procedure [9]. From Eq. 9 it is evident that there are $4 P$ coefficients, $P$ of which depend only upon composition $c$, and ' $3 P$ of which depend upon both $g$ and $c$. The inversion required by the left hand of Eq. 1 therefore forms a non-linear relationship with the $4 P$ microstructure variables. These microstructure variables lie in the associated $4 P$-dimensional subspace of the full (infinite-dimensional) space representing all possible local state distribution functions. Bounding this relationship is a hypersurface of the form $\langle C\rangle_{1111}^{-1}=S_{1111}^{*}$ residing in this same subspace. (The reader should note that components of the local state distribution function, not lying in the relevant $4 P$ dimensional subspace of the elastic property, have no relevance to the property of interest. This is typical in our experience - homogenization relationships such as Eqs. 1 \& 7 depend only upon components of microstructure in subspaces of modest dimension.)

In similar fashion, $\left\langle\lambda^{*}\right\rangle$ required in Eq. 7 can be expressed by the finite series

$$
\left\langle\lambda^{*}\right\rangle \approx \sum_{l=0}^{L} \sum_{\mu=1}^{M(l)} \sum_{n=0(2)}^{+l} \sum_{p=1}^{P} F_{l}^{\mu n} Y_{l}^{\mu n}\left(\partial_{11}\right)
$$

where ${ }_{p} Y_{l}^{\mu n}\left(\hat{\sigma}_{11}\right)$ are coefficients associated with the Sachs estimate for fcc metals subject to $\{111\}<110>$ slip. (It has been our experience that truncation at $L \leq 18$ provides sufficient resolution in the yielding relationship.) Eq. 10 defines a hyperplane lying in a finite dimensional subspace of the infinite-dimensional Fourier space. The coefficients ${ }_{p} Y_{l}^{\mu n}\left(\hat{\sigma}_{11}\right)$ define the normal to this hyperplane. All microstructures with Fourier coefficients, ${ }_{p} F_{l}^{\mu n}$, satisfying Eq. 10 are predicted to have $\sigma_{11}^{y}$ of at least that given by Eqs. $7 \& 10$, although Sachs model does not provide a rigorous bound. The limit planes described by Eq. 10 have been called iso-property hyperplanes [2,3].

\section{Determination of the Microstructure Hull}

It remains to describe the limitations of microstructure in the Fourier space, since the homogenization relations described previously do not place any limitations on the microstructure coefficients, ${ }_{p} F_{l}^{\mu n}$. To proceed, transform $H$ into an equivalent space, $H_{f}$, by expressing each possible local state, say $h_{j}$, as a Dirac function $\delta\left(h-h_{j}\right)$, with Fourier coefficients

$$
{ }_{p} F_{l}^{\mu-1}=\dot{\bar{T}}_{l}^{* \mu n}\left(g_{j}\right) \chi_{p}\left(c_{j}\right) \text {. }
$$

All possible microstructures pertinent to the selected homogenization relations can then be expressed as a sum of these Dirac functions weighted by appropriate volume fractions, $v_{j}$ :

$$
{ }_{q} F_{l}^{\mu n}=\sum \nu_{j} \dot{\dot{T}}_{l}^{* \mu n}\left(g_{j}\right) \chi_{q}\left(c_{j}\right)
$$

Relation (12), when considering all possible distributions on $H$, is the microstructure hull. It is compact and convex and it represents all possible microstructures within the selected mechanical framework. Intersections of the subset of microstructures defined by Eqs. $1 \& 9$, in the case of the lower-bound on elastic compliance $S_{1111}^{*}$, or the subset defined by relations Eqs. $7 \& 10$, in the case of the (pseudo) lower-bound on $\sigma_{11}^{y}$, with the microstructure hull defined by Eq.12, describe all possible microstructures satisfying the bounding relationships. These intersections are finite, according to the 
finite dimensional subspaces in which the homogenization relationships exist. This is depicted in Fig. 1 for a selected 3-dimensional microstructure subspace.

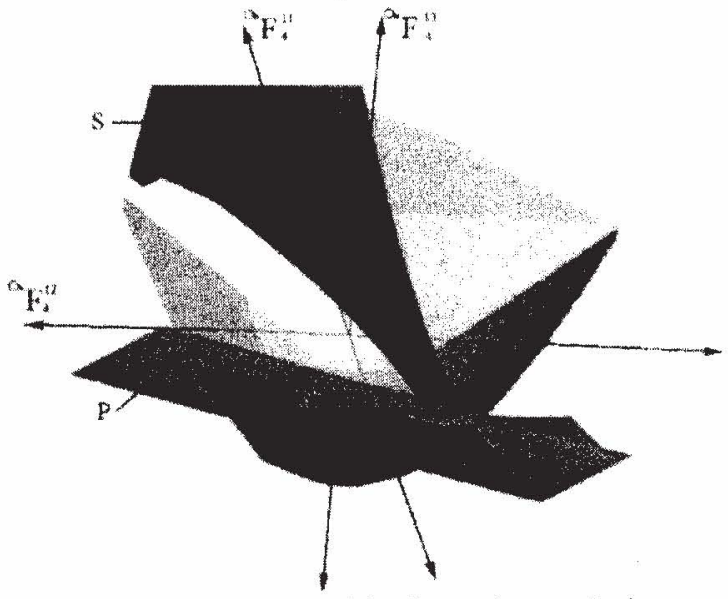

Fig. 1 Microstructure subspace with a hyperplane and a hypersurface depicting microstructure properties

\section{Recovery of the Properties Closure}

The sets of all possible property combinations predicted by the theory can be obtained by sampling the microstructure hull. When the homogenization relations are simple arithmetic averages of microstructure components, as is the case with the Sachs (pseudo) lower-bound, or the upper bound on $S_{1111}^{*}$, which is given by the right hand side of Eq. 1, a systematic consideration of the intersections of the family of finite-dimensional iso-property hyperplanes for each property of interest with the convex microstructure hull yields all possible property combinations. The bounds of the closure can be obtained by performing a min-max linear optimization analysis [4].

In this paper, one of the properties of interest is the lower-bound on $S_{1111}^{*}$, and the traditional linear methodology is insufficient. A nonlinear solution can be efficiently approximated by using a Monte Carlo method. Here groups of two local states were selected randomly, represented by Fourier points ${ }_{p} F_{l}^{\mu n}(1)$ and ${ }_{p} F_{l}^{\mu n}(2)$. Microstructures lying upon the line connecting these two points are then considered according to the formula

$$
{ }_{p} F_{l}^{\mu n}=v_{1 p} F_{l}^{\mu n}(1)+v_{2 p} F_{l}^{\mu \mu_{i}}(2),
$$

subject to the fundamental constraints $\sum v_{j}=1$ and $v_{j} \geq 0$. Approximately twenty different volume fraction combinations were considered for each Monte Carlo step.

Property combinations were calculated using the lower-bound homogenization relations. The locus of all combinations is the estimate of the properties closure, which is shown in Fig. 2. The property closure can be more precisely determined by nonlinear optimization techniques including generalized reduced gradient and sequential quadratic programming methods.

Extension of the Monte Carlo this method to more complex microstructures, resulting from mixtures of more than two components, is found to have negligible impact in extending the closure. Since the extremal combinations of properties, those that lie on the periphery of the properties closure, are typically associated with single local state microstructures, these are naturally included in the Monte 


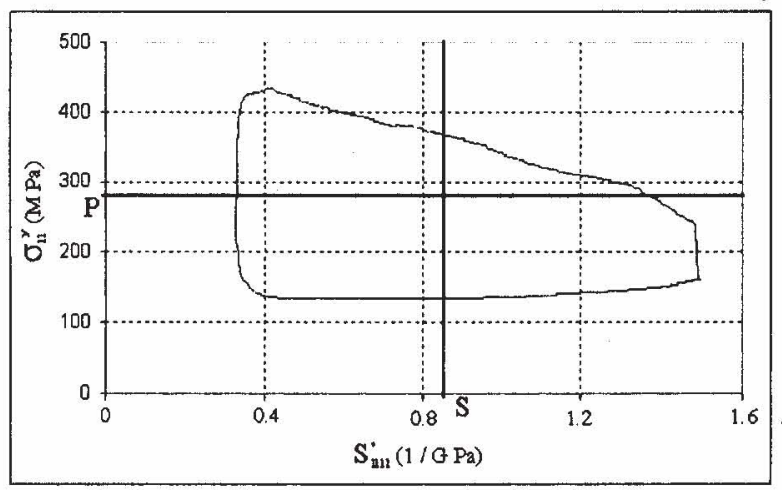

Fig. 2 Properties closure for $\mathrm{Ni}-\mathrm{Cu}$ System showing design constraints and feasible solution area

Carlo simulation procedure used.

\section{Conclusions}

The methodology described in this paper provides a framework for obtaining the theoretical (combined) properties closure where the relevant homogenization relationships are non-linear. In this case, the construction of the combined properties closure cannot rely upon classical min-max algorithms in linear analysis. We have obtained reliable estimates of this closure via a Monte Carlo procedure although it requires considerably more computational effort than was required in the linear case.

\section{Acknowledgement}

Support of the Army Research Office is greatly appreciated.

\section{References}

[1] H. J. Bunge, Texture Analysis in Materials Science, Butterworths (1982) p. 347.

[2] B. L. Adams, A. Henrie, B. Henrie, M. Lyon, S. R. Kalidindi, and H. Garmestani, J. Mech. Phys. Sol. 49 (2001) p. 1639-63.

[3] B. L. Adams, M. Lyon, B. Henrie and S. Kalidindi, in Proceedings of Army Sagamore Conference, ed. E. Chin (2001) to appear.

[4] B. L. Adams, M. Lyon, B. Henrie, S. R. Kalidindi and H. Garmestani, in Plasticity, Damage and Fracture at Macro, Micro and Nano Scales, eds. A. S. Khan and O. Lopez-Pamies, Neat Press, MD (2002) p. 3-5.

[5] R. Hill, Proc. Phys. Soc. 65, (1952) p. 349.

[6] B. Paul, Trans. Metall. Soc. AIME, 218 (1960) p. 36-41.

[7] G. Sachs, Z. Ver. Dtsch. Ing. 72 (1928) p. 734-36.

[8] J. P. Keener, Principles of Applied Mathematics, $2^{\text {nd }}$ Ed., Perseus, Cambridge, Mass. (2000) p. 82.

[9] J. P. Hirth and J. Lothe, Theory of Dislocations, McGraw Hill, New York (1968) p. 398. 